\title{
Metaphorical Subject Nomination of Non-spatial Spheres (based on the English language)
}

\author{
V. V. Yanina \\ Department of Foreign Language Communication \\ Volgograd State University \\ Volgograd, Russia \\ yanina230172@volsu.ru \\ N. B. Egorchenkova \\ Department of Germanic and Romance philology \\ Volgograd State University \\ Volgograd, Russia \\ Natalia.egorchenkova@volsu.ru
}

\author{
L. N. Rebrina \\ Department of Germanic and Romance philology \\ Volgograd State University \\ Volgograd, Russia \\ LNRebrina@volsu.ru \\ E. A. Eltanskaya \\ Department of Foreign Language Communication \\ Volgograd State University \\ Volgograd, Russia \\ yeltanskaya@volsu.ru
}

\begin{abstract}
The article presents the results of the spatial metaphor as a way of understanding and nomination of objects and phenomena of directly not observed social, mental and intellectual spheres study. The authors regard the status and the functional importance of nouns denoting limited space for these processes. In the article, the set of English polysemantic spatial nouns denoting limited space are described, semantic multipliers in their basic meaning are revealed, the metaphorical and axiological meanings of polysemants are defined. The authors consider semantic modifications of spatial nouns and describe the models of metaphorical transfers on the basis of re-thinking spatial features. As it is realized, the study results show that in most regular metaphorical models, the metaphorical transfer of spatial characteristics onto the events and phenomena of social sphere is the most frequent. The metaphorical transfer of spatial characteristics onto the mental phenomena is marked by lower frequency, and as a result it is realized in a smaller number of metaphorical models. The metaphorical transfer of spatial characteristics onto intellectual sphere is marked by the lowest frequency. The authors reveal the spatial features which prove to be essential in the evaluation process. The article shows that from a great number of semantic multipliers which characterize limited fragment of space, the following spatial features are actualized: 'size', 'border', 'coordination parameters', 'configuration', 'filling', 'functional characteristics', 'constituents', and 'structural characteristics', 'functional characteristics'. Examples illustrating the functioning of the studied lexical units in the contexts are given.
\end{abstract}

Keywords - lexical unit, semantics, space, metaphorical model, metaphoric transfer, evaluation.

\section{INTRODUCTION}

The research is devoted to metaphorical modeling of nonspatial spheres on the basis of reinterpretation of semantic signs of spatial nouns in modern English. The choice of this subject is caused by the fact that space is one of the basic, universal categories of reality perception, receiving reflection in the language, which is an "integral component of a chain of interactions among man, society, nature" [1]. And there is experience of interaction of the native speaker with the physical world as a result of cognitive and language activity of the man. The spatial metaphor represents the effective cognitive mechanism of mental and language development of reality [2]. Within the conducted research, while revealing regularities of spatial objects representation in English, the cognitive approach, allowing one to determine features of space perception by native speakers of this language which are fixed in semantics of the corresponding lexical units, is implemented and mediates interpretation and nomination of the fragments of the world around belonging to both spatial and non-spatial spheres [3]. The study object is English polysemantic nouns of spatial semantics, the subject semantic signs in the structure of primary and secondary meaning of the specific nominative units, objectifying conceptual integration of spatial and non-spatial spheres. The research relevance is determined by the need of studying units of the of spatial objects nomination in English for the purpose of identification of signs, relevant for conceptualization and language denotation of physical space fragments, and metaphorical models, the assessment mechanism upon the figurative nomination of elements of non-physical space. The theory of the metaphor is an effective methodological tool of modern linguistics, and a space metaphor - one of the most widespread language structures. Despite the importance and prospects of the last, separate aspects of this area of research remain insufficiently studied. The results can be used to solve practical problems of linguodidactics, lexicology, translation, lexicography, cultural linguistics.

\section{MethodologY}

Methodological bases of the research are: works devoted to a cognitive function of the language [4-6], linguistic modeling [7-9], development of the theory of a metaphor as the mechanism of mental and language development of reality [10-13]. In the work, the conceptual framework, developed taking into account achievements of modern linguistics in the 
field of spatial semantics [1, 5, 14-21]. In addition, the characteristics of physical space selected by domestic and foreign linguists, the available classifications of lexical units with spatial semantics and the methodological principles of the theory of a metaphor are considered.

The research material is the most frequency multiplevalued nouns of modern English with spatial semantics (90 lexical units) which are selected by way of continuous selection of lexicographic sources (Compact Oxford English Dictionary (COED), Longman Dictionary of Contemporary English (LDCE), Collins English Dictionary (CED), Cambridge Advanced Learner's Dictionary (CALD), Merriam-Webster Online Dictionary (M-WOD)); the contexts (3000) illustrating functioning of the studied lexical units, taken from modern different genres works of art and publicist prose of English-speaking authors. Despite a large number of works in modern linguistics devoted to the general description of the category of space (Yu. D. Apresyan, E. S. Yakovleva), studying its separate aspects, such as language means and ways of expression of spatial localization (N. L. Shamne, A. D. Shmelyov), semantics of spatial names (A.A. Ufimtseva, E. V. Rakhilina), spatial pretexts and adverbs (M. V. Vsevolodova, T. N. Malyar, O. N. Seliverstova), a spatial metaphor (T. N. Malyar, O. N. Seliverstova, V. N. Toporov), the English polysemantic spatial nouns in their basic, metaphorical and attitudinal meanings have not been considered entirely so far. In the research, the polysemantic spatial nouns corpus is defined, semantic multipliers in their major meaning are singled out, metaphorical and attitudinal meaning of polysemants is determined. Besides, semantic modifications of spatial nouns are considered, models of metaphorical transfers on the basis of reconsideration of spatial signs are described, connection of spatial signs with the sphere of an assessment is established, spatial signs which are actual in the course of estimation are analyzed. For the solution of the declared research problems along with the general scientific methods of observation and description (generalization, systematization and tipologization), methods of the component, definitional and contextual analysis, linguistic methods of the definitional, component, contextual analysis, metaphorical modeling and quantitative calculations are used. To solve the tasks of the project, the authors:

1) define a paradigm of the English nouns denoting spatial objects, reveal the structure of this lexical subsystem and degree of lexical representation of different types of space fragments;

2) describe functional and semantic signs of LSV of the revealed nouns used for the nomination of objects of physical space; select the relevant characteristics indicating specifics of perception of space by representatives of the English-speaking linguoculture, which are reflected in the direct meanings of lexical units;

3) allocate LSV of polysemant with secondary meanings, define dominant spheres of metaphorical transfer and signs motivating it;

4) reveal evaluative components in the structure of primary and secondary meanings of English spatial nouns;
5) characterize mechanisms of formation of nouns figurative meanings, define basic models of the metaphorical nomination of polytypic objects on the basis of spatial signs.

The internal organization of the studied paradigmatic association of polysemants corresponds to ontological types of spatial objects and is constituted by two groups: 1) natural limited fragments of space; 2) anthropogenous limited fragments of space. The first group of nouns is lexically most fully presented that shows the importance of the nominated spatial objects for the English-speaking lingvoculture and higher metaphorical potential of the corresponding lexical units. The structural elements of this group are subgroups "the space filled with terrestrial substance", "space, filled with water substance" and "the space filled with air substance" and microgroups forming them. In both groups, the lexemes denoting fragments of a landscape, rooms of different function, objects and devices for implementation of any activity, the characteristic of a form of a subject, a way of movement, a part of the body, and also nouns with generally spatial semantics are the most numerous.

\section{RESUltS}

The authors analyze a subset of the lexical units (LU) relating to the lexical-semantic group (LSG) of spatial nouns which semantic macrostructure includes more than one lexicosemantic variant (LSV) that is the polysemantic spatial nouns. LSG of spatial nouns considered is constituted by the words of one part of speech on the basis of the integrating component of a lexical meaning of a group words (categorical and lexical seme 'limited fragment of space') and forms the paradigmatic field with a certain internal organization.

LSV of a spatial noun represents a nominative unit in one of its lexical meanings as it is described in defining dictionaries, which set forms a semantic macrostructure of a multiple-valued constituent.

The authors limited a framework of their analysis to the polysemantic nouns with the specified component of meaning specialized on the nomination of spatial objects and metaphorical naming of fragments of non-spatial spheres, regularly participating in generation of statements.

The semantic structure of LSV of polysemantic spatial nouns in their direct meaning consists of the semantic signs actualized within the following semantic multipliers in the basic meaning of LE: "filling a limited fragment of space" where there is a reference to the degree and the nature of filling the space (lack of objects, full filling, not in full filling, filling by homogeneous/heterogeneous objects, filling by static/dynamic objects). The semantic multiplier "distance from the observer" unites the lexical units in the basic meaning naming the size of an interval between objects (considerable/insignificant distance, lack of distance between objects). The semantic multiplier "coordination parameters of a limited fragment of space" is lexemes, in basic meanings of which such semantic signs as reference to find coordinates on an axis are realized (bottom, top, center, periphery, external space, internally space, the absolute/relative characteristic of coordinates of a limited fragment of space, vertical position, horizontal position, front situation). The semantic multiplier 
"the size of a limited fragment of space" unites lexemes, in basic meaning of which the semantic signs naming the extent of height, depth, length, considerable / insignificant volume are realized. The semantic multiplier "configuration of a limited fragment of space" is realized in the meaning of lexical units by the semantic signs, which refer to a form (point, line, circle, square). The semantic multiplier of the "border of a limited fragment of space" is realized in the meaning of lexical units by the semantic signs having the reference to the function of a spatial limit (presence / lack of restriction; distinct/indistinct clearness of the border, mobile / static character of the border). The semantic multiplier "the origin of a limited fragment of space" is realized in the meaning of lexical units by semantic signs 'anthropogenic / man-made', 'natural/not made by hand'. The semantic multiplier "the functional characteristic of a limited fragment of space" is realized in the meaning of lexical units by semantic signs 'protective function', 'focusing function', 'esthetic function' etc. The semantic multiplier "the structural characteristic of a limited fragment of space" is realized in the meaning of lexical units by semantic signs 'a part the whole', 'isolation', 'openness', 'gradualness', etc. The semantic multiplier "character of the constituting material" is realized in the meaning of lexical units by semantic signs 'strong', 'specific', etc. The semantic multiplier "subjective assessment of a limited fragment of space" is realized in the meaning of lexical units by semantic signs 'positive', 'negative'. Let us assume this classification with modifications as a basis when describing and systematizing the actual material. Figurative meanings of spatial nouns reflect social, intellectual and mental fields of man's activity. At the same time, the following metaphorical transfers have regular character. Within the social sphere - "space $\rightarrow$ a socio-political situation", "space $\rightarrow$ moral and ethical beliefs", "space $\rightarrow$ socio-professional activity", "space $\rightarrow$ welfare activity", "space $\rightarrow$ social and legal activity", "space $\rightarrow$ the social characteristic of an anthropogenic spatial object". Within the mental sphere - "space $\rightarrow$ an emotional state", "space $\rightarrow$ psychological influence", "space $\rightarrow$ interpersonal relations", "space $\rightarrow$ mental characteristic of the subject", "space $\rightarrow$ emotional characteristic of an anthropogenic spatial object". Within the intellectual sphere - "space $\rightarrow$ intellectual activity", "space $\rightarrow$ mental characteristic of the subject". The structure of the analyzed lexical-semantic variants of multiplevalued nouns is constituted by a set of the semantic multipliers realized by concrete semantic signs.

The greatest conceptual contiguity with the spatial sphere in mental projections of native speakers of English is characteristic of social and mental spheres. For the figurative nomination of these objects of the spheres, such semantic multipliers are general and significant as: "filling a limited fragment of space"; "distance from the observer"; "coordination parameters of a limited fragment of space"; "border of a limited fragment of space"; "the size of a limited fragment of space"; "the structural characteristic of a limited fragment of space"; "the functional characteristic of a limited fragment of space".

Within the considered spheres, the highest nominative potential is characteristic of metaphorical transfer "the spatial sphere $\rightarrow$ the social sphere". The metaphorical nomination of objects of the intellectual sphere on the basis of analogy to fragments of space is least relevant for the English linguoculture.

Multiple-valued nouns which the semantic structure, finding metaphorical transfer of spatial characteristics by the phenomena of the mental sphere, are also frequent in English and also realize the following regular metaphorical models: "space $\rightarrow$ man's emotional state", "space $\rightarrow$ psychological effect", "space $\rightarrow$ interpersonal relations", "space $\rightarrow$ mental characteristic of the subject", "space $\rightarrow$ emotional characteristic of an anthropogenous spatial object".

The spatial nouns used for metaphorical nomination of the phenomena of the intellectual sphere are least presented in a quantitative sense. The following metaphorical models are regular: "space $\rightarrow$ intellectual activity of the person", "space $\rightarrow$ mental characteristic of the subject".

The results of the carried-out component and contextual analysis of the entire massif of the language facts have shown that in $12.2 \%$ cases, derivative LSV of spatial nouns remains the means of nomination within the spatial sphere, but gains properties of an evaluative language unit and can serve as means of expression of the subjective relation to a nomination object. In these LSV, the spatial signs reflected in such semantic multipliers as a "size of a limited fragment of space", a "border of a limited fragment of space", a "configuration of a limited fragment of space", "filling a limited fragment of space", "functional characteristic of limited space fragment", "constituents of a limited fragment of space", "coordination parameters of limited space fragment" and "the structural characteristic of a limited fragment of space" are currently central in the course of evaluation.

Let us consider one of examples of realizing the model of metaphorical transfer "the space sphere $(\mathrm{SpS}) \Rightarrow>$ the social sphere".

The noun jungle $_{1}$ has the direct spatial meaning "dense tropical forest with many large plants and tangled vegetation"[22]. The semantic structure of LSV jungle I $_{I}$ is set up by semantic signs 'natural', 'the maximum filling', 'filling by diverse objects', 'insignificant distance between objects', 'having a kinking form', 'setting back movement' which have formed the basis for formation of figurative meanings of a considered noun: jungle ${ }_{2}$ "a situation in which it is difficult to succeed or get what one wants because a lot of people are competing against each other"; jungle j $_{3}$ "something that is very complicated, or confusing".

On metaphorical reconsideration of spatial characteristics in the semantic structure of a noun, the signs reflecting specifics of space filling - the degree and the nature of filling - are actualized. In the social sphere, excessive space filling is comprehended as a big competition, and the nature of filling as something tangled and difficult.

The semantic structure of LSV jungle ${ }_{2}$ has a speaker's negative assessment of a nomination object. It becomes obvious in the semantic analysis of such components of interpretation as "difficult to succeed or get what one wants", "a lot of people are competing against each other". The 
semantic structure of LSV jungle 3 also has a speaker's negative assessment of a nomination object that is confirmed by the semantic analysis of such components of interpretation as "something that is very complicated, or confusing". The transfer on the phenomena of the social sphere of man's activity has the following spatial signs: 'maximum filling', 'filling by heterogeneous objects', 'setting back movement', 'insignificant distance between objects', 'having a kinking form' is observed. For example: (1) It's hard to succeed in the business world. It's a jungle out there [23]; (2) A jungle of environmental laws baffles local community [24].

In the context of (1), the noun jungle $e_{2}$ defines the place of fight for existence under conditions of the big competition. The model of metaphorical transfer "space $\rightarrow$ man's socialprofessional activity (SPAS )" is realized: $\mathrm{SpS} 1$ 'the maximum filling', 'filling by heterogeneous objects', 'setting back movement', 'insignificant distance between objects' (EVN (evaluation) + (positive) - (negative) $/+-($ ambivalent $) /+-$ /+-); SPAS 'competition existence', 'difficult', 'tangled' (EVN $-/-1-)$.

In context (2), the noun jungle 3 is used for the negative characteristic of a spatial object - the jungle which in the analyzed context is defined as "confusion, a tangled situation". The model of metaphorical transfer "space $\rightarrow$ a social-political situation (SPSS)" is realized: SpS 'full filling', 'filling by heterogeneous objects', 'insignificant distance between objects', 'having a kinking form' (EVN +-/+-/+-/-); SPSS is 'difficult', 'tangled', 'confusing' (EVN -l-l-).

Let us consider one of examples of realization of the model of metaphorical transfer "the spatial sphere $\Rightarrow$ the mental sphere. LSV cocoon $_{l}$ is defined as "the covering made of soft smooth threads that surrounds and protects particular insects during the pupa stage as they develop into the adult form" [22]. The component of interpretation "the covering made of soft smooth threads that surround and protect particular insects" contains the reference to "the origin of a limited fragment of space", LSV is realized in this by semantic sign natural / not of human making'; the component of interpretation "made of soft smooth threads" indicates a semantic multiplier "quality composition of the constituting material of a limited fragment of space", realized by semantic signs 'specific material', 'soft'. The component of interpretation "during the pupa stage" refers to a presence of a semantic multiplier "existence period of a limited fragment of space" in the structure of LSV $\operatorname{cocoon}_{l}$, objectified by the semantic sign 'limited in time'. Within a semantic multiplier "coordinating parameters of a limited fragment of space", the component of interpretation "that surrounds" notes in this LSV a semantic sign 'frontal arrangement' (what is outside of the entire and fences). The component of interpretation "protects" is indicated by a semantic multiplier "the functional characteristic of a limited fragment of space", realized by a semantic sign 'protective function'. The components "that surrounds" and "covering" contain the reference to a semantic multiplier "the structural characteristic of a limited fragment of space" and allow one to mark out semantic signs 'a part of the whole' (a cocoon - the cover, i.e. a part of difficult formation in which the case-worm of an insect develops, and this cover (a covering) surely assumes filling).
For example: (3) Indeed the silk, the most luxurious of all our fabrics, is unwound from the cocoon that silk-moth caterpillars weave around themselves before they start the complex process of changing into adults. Actually, silk, the most magnificent of all fabrics, is obtained from a cocoon which caterpillars of a silkworm bind around themselves before they begin a difficult process of turning into an adult individual [24]. The given example illustrates such semantic signs expressed in the definition as 'natural / not made by hand' 'part of space' (the cocoon that silk-moth caterpillars weave), 'limited in time' (the complex process of changing into adults), 'frontal arrangement', 'protective function', 'a part of the whole' (cocoon that silk-moth caterpillars weave around themselves).

Let us analyze a definition of a component of the dictionary interpretation of LSV cocoon $_{1}$ - "covering" - "a layer of something that covers or hides or protects something" [22]. It is the layer from something covering, hiding or protecting something) which will allow one to increase the depth of defining and to verify the revealed semantic signs of LSV $\operatorname{cocoon}_{1}$, relevant for the subject spatial nomination: 'protective function', 'frontal arrangement', 'a part of the whole'. The component of interpretation "covering a layer of something" refers to a semantic sign 'a part of the whole'; a component of interpretation «that covers or hides or protects something» - to a sign 'protective function'. Thus, by results of the analysis of dictionary definitions and the contextual analysis, the semantic structure of LSV $\operatorname{cocoon}_{1}$ is constituted by the following semantic signs: 'a natural / not of human making' part of space, 'limited in time', 'a frontal arrangement', 'protective function', 'a part of the whole, 'specific material', 'soft'. The above-mentioned semantic signs formed the basis for formation of a figurative meaning - LSV of cocoon $_{2}-\mathrm{a}$ "place or situation in which one feels comfortable and safe, and are protected from anything unpleasant" [25] - the place or situation where one feels comfortable and safe and is protected from all troubles. The semantic sign of LSV cocoon $_{l}$ 'soft' is transformed to a sign 'pleasant' in the structure of LSV cocoon $_{2}$ (denotation through a component of interpretation "in which one feels comfortable"), the sign of cocoon $_{l}$ 'limited in time' makes it possible to transfer the nomination from the sphere of the spatial relations to the sphere of the temporal relations (a component of interpretation "situation"); the semantic sign of cocoon $_{l}$ - 'protective function' remains in semantic structure of cocoon $_{2}$ (denotation through components of interpretation "safe", "protected from anything unpleasant"). In the semantic structure of this unit, the reference to the relevance of a semantic sign "positive assessment" of a nomination object is present, which is traced in the semantic analysis of the components of interpretation "comfortable" and "safe".

Thus, one can observe a transfer of spatial signs to the objects and the phenomena of the mental sphere, as a result of which the spatial noun cocoon takes on a figurative meaning and is used for the nomination of emotional states. For example: (4) He had rolled through childhood in a warm cocoon of love provided by endless cousins, uncles, aunts and servants [25]. In the given context, LSV cocoon $_{2}$ is used for qualification of an emotional state of the person during a 
certain period of time - loving environment of the family. In the analyzed context, the lexical unit cocoon is represented as a certain existential localization of the subject in which he/she feels protected, guarded that makes living pleasant, comfortable; (5) Now I would like to crawl back into my cocoon of bedclothes and sleep and sleep [26]. In this example, LSV cocoon $_{2}$ characterizes an emotional state of the speaker, additional qualification of an object of the nomination - bed linen, which is interpreted by the subject as the certain intimate shelter, a part of space providing comfort, privacy and rest.

The analysis allows drawing a conclusion on relevance of a number of spatial signs for the nomination of non-spatial (mental) sphere objects. In the given example of metaphorical transfer, the "space $\rightarrow$ emotional state" model is implemented: $\mathrm{SpS}$ 'protective function', 'closed', 'a part of the whole', 'specific material' (soft), 'limited in time' (EVN +/+-/+/+-/+-) $\Rightarrow$ ESS (emotional state sphere) 'providing privacy, rest', 'pleasant', 'comfortable', 'a certain period of time' (EVN $+/+/+/+-)$.

Let us consider one of examples of realizing the model of metaphorical transfer "the spatial sphere $\Rightarrow>$ the intellectual sphere". LSV mug $_{1}$ has the direct spatial meaning: "a large cup, typically cylindrical with straight sides, with a handle, made of earthenware and used for hot drinks" [23]. The analysis of a dictionary definition will allow us to carry out decomposition of a lexical meaning and to allocate the explicit semantic multipliers constituting the semantic structure of the lexical unit and which are implemented in concrete semantic signs. Components of interpretation "cylindrical", "with straight sides" refer to a semantic multiplier "a configuration of a limited fragment of space", realized in the semantic structure meaning of this LSV by semantic signs 'cylindrical', 'equilateral'. The component of interpretation "large" refers to a semantic multiplier "the size of a limited fragment of space" realized in the semantic structure meaning of this LSV by semantic sign 'considerable'. The component of interpretation "made of earthenware" refers to a semantic multiplier "the qualitative structure / characteristic of a limited fragment of space" realized in the semantic structure meaning of this LSV by semantic sign 'clay'. For example: (6) He was using a real coffee mug he had brought down from the Open-Unsolved Unit because he preferred it over Styrofoam [25]; (7) The gin was served out to them in handleless china mugs [24]; (8) I made myself a large mug of cocoa [22]. The given examples illustrate semantic signs explicated in the definition: 'clay', 'considerable' (size) (china mugs, a real coffee mug, a large mug of cocoa).

Thus, by results of the analysis of dictionary definitions and the contextual analysis, the semantic structure of LSV $\mathrm{mug}_{1}$ is constituted by the following semantic signs: 'cylindrical', 'equilateral', 'considerable' (size), 'clay'. These semantic signs formed the basis for formation of a figurative meaning of LSV $\mathrm{mug}_{2}$ : «a person who is stupid or gullible and easily deceived» [23]. During transfer to the intellectual sphere semantics, a form and qualitative structure of a limited fragment of space is important for the English native speaker. The subject having the cylinder form, the equal parties, made of material, which under insignificant influence takes any form easily, is metaphorically comprehended as a silly, trustful, naive person. Thus, during transfer, spatial semantics of a form is actualized. The semantic structure of the unit contains the reference to the negative assessment of a nomination object that it is traced in the semantic analysis of such components of interpretation as: "stupid, gullible and easily deceived", and a transfer of spatial signs: 'cylindrical', 'equilateral', 'considerable' (size), 'clay' - to an intellectual field of man's activity is also observed. For example: (9) $\mathrm{He}^{\prime} \mathrm{s}$ such a mug, he believes everything she tells him [24]; (10) They were no mugs where finance was concerned [22]. In these examples, LSV $\mathrm{mug}_{2}$ is used for the negative characteristic of a certain spatial object - mug, which in the analyzed context is defined as the trustful, naive, silly person who is easy to deceive, and transformation of a spatial object to an object of the intellectual sphere is shown. The contextual analysis confirms the authors' conclusion on the relevance of the spatial signs for the nomination of objects of non-spatial (intellectual) sphere which were marked out.

In this example of metaphorical transfer, the "space $\rightarrow$ mental characteristic of the subject (MCSS)" model is implemented: $\mathrm{SpS}$ is 'cylindrical', 'equilateral', 'considerable' (size), 'clay' (EVN 0/0/0/0) $\Rightarrow$ MCSS 'trustful', 'naive', 'simple', 'silly' (EVN-/-/-/-).

\section{CONCLUSION}

The polysemantic nouns defining limited fragments of space plays a significant role in the process of the subject nomination. They form the paradigmatic group of lexical units entering the semantic field "Space" as the constituting element. The analysis of semantic modifications of spatial nouns has shown that constituents of this group are capable to express a wide range of meanings - from basic spatial to the figurative ones, reflecting native speakers' ideas of objects (phenomena, events, situations) of social, mental, intellectual spheres of man's being. The semantic signs are present in the structure of the direct meaning; the polysemantic spatial nouns form the basis for formation of figurative meanings, in which the value judgment, formed in the mind of English-speaking society representatives, is expressed. The spatial signs which are contained in the LSV structure of the nouns with spatial semantics are relevant for the metaphorical name of the objects of mental, intellectual and social non-spatial spheres and cause formation of evaluative components in the semantic structure of figurative meanings. There are such spatial signs relevant in the course of estimation as: 'size', 'border', 'configuration', 'filling', 'functional characteristic', 'constituents', 'coordination parameters' and 'structural characteristic' of limited fragments of space. Thus, it is possible to note that evaluation of space was formed in the course of man's activity and his knowledge of the world.

The metaphor as the cognitive mechanism mediates the knowledge of objects directly inaccessible to understanding of objects and the phenomena of social, mental and intellectual spheres. Metaphorical modeling of objects of non-spatial spheres on the basis of spatial signs allows revealing the nature and significant parameters of space perception and images of the semantic spheres arising on the basis of 
understanding the space. Within the conducted research, while revealing regularities of spatial objects representation in English, the cognitive approach allowing one to determine features of space perception by native speakers of the language, which are fixed in semantics of the corresponding lexical units, is implemented and it mediates interpretation and nomination of the fragments of the world around belonging to both spatial and non- spatial spheres.

\section{References}

[1] N.L. Shamne, "The field "space" in the Russian and German languages (culturological aspects of the linguistic analysis of texts," "Problems of speech communication", Saratov: Izd-vo Sarat. Un., 2000. pp. 43-50.

[2] Dzh. Lakoff, M. Johnson, "Metaphors We Live By" In: "Language and Modelling of Social Interaction", Moscow, Progress Publ., 1988. (in Russian).

[3] N. Shamne, A. Petrova, L. Rebrina, M. Milovanova, "Phenomenon of memory and category of space: Gnoseological and communicative status," "XLinguae," vol. 7, Iss. 3. pp. 32-49, 2014.

[4] W. Xie, W. Zhang, "Contributions of Cognitive Factors in Conceptual Metaphors," "Metaphor and Symbol," 29(3), pp.171-184, 2014. (in Russian).

[5] R. Jakendoff, B. Landau, D. Napoli "Spatial Language and Spatial Cognitionm", "Bridges between Psychology and Linguistics. A Swarthmore Festschrift for Lila Gleitman Hellsdole”, New Jersey, 1991, pp. 145-169.

[6] K. Stocker, "The Theory of Cognitive Spacetime," "Metaphor and Symbol", vol. 29(2), pp.71-93, 2014.

[7] L. Talmy, „How Language Structures Space,” “Spatial Orientation. Theory, Research and Application," N.Y., L.: Plenum Press, 1983. pp. 225-282.

[8] L. Talmy, "Lexicolization patterns: Semantic Structure in Lexical Forms,". In: T. Shopen "Language Typology and Syntactic Description”, 3. Cambridge University Press, 1985. pp. 57-149.

[9] E.S. Yakovleva, "Fragments of the Russian language picture of the world (model of space, time and perception)," Moscow: Gnozis, 1994. (in Russian).

[10] E.M. Wolf, "A metaphor and assessment," "Metaphor in language and text”, Moscow, 1988, pp. 52-65. (in Russian).
[11] B.E. Vladimirovich, C.A. Prokopievich, T.G. Filippovna, "Conceptual metaphor in educational discourse," "Biosciences Biotechnology Research Asia," 12. (1). pp. vol. 561-567, 2015.

[12] E.E. Anikin, E.V. Budaev, A.P. Chudinov, "Historical dynamics of metaphoric systems in Russian political communication," "Voprosy Kognitivnoy Lingvistiki”, vol. 3 (44). C. 26-32, 2015.

[13] M. Domínguez, "On the Origin of Metaphors," "Metaphor and Symbol," 30(3), pp.240-255, 2015.

[14] M.V. Vsevolodova, "Ways of expression of the spatial relations in modern Russian,” Moscow: Russian, 1982. (in Russian).

[15] E.S. Kubryakova, "About concepts of the place, subject and space In: "Logical analysis of language. Languages of spaces," Moscow: Languages of the Russian culture, 2000, pp. 84-92. (in Russian).

[16] T. N. Malyar, "Conceptualization of space and semantics of the English spatial pretexts and adverbs," PhD Thesis, Moscow, 2002. (in Russian).

[17] T.N. Malyar, "Semantics of some spatial pretexts in the Russian and English languages," In: T.N. Malyar, O.N. Seliverstova, "Contrastive Linguistics". Sofia, T. XVII, No. 3. pp. 118-123, 1992. (in Russian).

[18] T. N. Malyar, "Spatial and remote pretexts and adverbs in the Russian and English languages," In: T.N. Malyar, O.N. Seliverstova, "VerlagOttoSagner". Munchen, 1998.

[19] Y. Lander, T. Maisak, E. Rakhilina, "Verbs of aquamotion: semantic domains and lexical systems (Book Chapter)". In: "Motion Encoding in Language and Space". 2013. pp. 67-83.

[20] N.L. Shamne, "The history of functioning of verbs of motion in comparative aspect," In: Translation and comparative linguistics. Yekaterinburg, 2004. pp 30-33.

[21] Shamne N., Rebrina L., Petrova A., Milovanova M., Eltanskaya E. "Space of memory: Interactional and semantic aspects," In: "Journal of Language and Literature,” vol. 5, Iss. 4, pp. 147-154, 2014. (in Russian).

[22] David Attenborough, "The trials of life" [Electronic resource]. Access mode: http://bookfi.org.

[23] MWOD - Merriam-Webster OnLine Dictionary. [Electronic resource] Access mode: http://www.m-w.com.

[24] CALD - Cambridge Advanced Learner's Dictionary. [Electronic resource]. - Access mode:.http://dictionary.cambridge.org.

[25] Colin McDowell, "A woman of style [Electronic resource]. Access mode:http://bookfi.org.

[26] COED - Compact Oxford English Dictionary. [Electronic resource ]. Access mode: http://oxforddictionaries.com. 